\title{
Oxidative Stress and Antioxidants in Athletes Undertaking Regular Exercise Training
}

\author{
Trent A. Watson, Lesley K. MacDonald-Wicks, \\ and Manohar L. Garg
}

\begin{abstract}
Exercise has been shown to increase the production of reactive oxygen species to a point that can exceed antioxidant defenses to cause oxidative stress. Dietary intake of antioxidants, physical activity levels, various antioxidants and oxidative stress markers were examined in 20 exercise-trained "athletes" and 20 age- and sex-matched sedentary "controls." Plasma $\mathrm{F}_{2}$-isoprostanes, antioxidant enzyme activities, and uric acid levels were similar in athletes and sedentary controls. Plasma $\alpha$-tocopherol and $\beta$-carotene were higher in athletes compared with sedentary controls. Total antioxidant capacity tended to be lower in athletes, with a significant difference between male athletes and male controls. Dietary intakes of antioxidants were also similar between groups and well above recommended dietary intakes for Australians. These findings suggest that athletes who consume a diet rich in antioxidants have elevated plasma $\alpha$-tocopherol and $\beta$-carotene that were likely to be brought about by adaptive processes resulting from regular exercise.
\end{abstract}

Key Words: oxidative stress, $\mathrm{F}_{2}$-isoprostanes, antioxidants, diet

Exercise has been shown to increase the production of reactive oxygen species (ROS) to a point that can exceed antioxidant defenses to cause oxidative stress (10). Oxidative stress has been linked to the pathogenesis of many chronic diseases (32) and has demonstrated links to fatigue (27), muscle damage (27), and reduced immune function (5), which can all affect exercise performance. Thus, there is a paradox between acute exercise increasing oxidative stress and regular exercise having beneficial effects on health and exercise performance. It is unknown why the exercise-oxidative stress paradox exists, but it has been hypothesized that the capacity and adaptation of the body's antioxidant defenses might be part of the reason (18).

A broad array of endogenous antioxidant defense mechanisms exist and various antioxidant nutrients are consumed in the diet that provide protection against the excessive production of ROS. Various adaptive processes have also been shown to take place in response to regular exercise that enhance or maintain antioxidant defenses. In general, endogenously produced antioxidant enzymes have been shown to up-regulate in response to exercise (16). The de novo production of endogenous antioxidant molecules such as urate have also been shown to increase

The authors are with the Discipline of Nutrition and Dietetics, Faculty of Health, University of Newcastle, NSW 2308, Australia. 
in response to exercise $(3,7,20,30,33)$ and mobilization of antioxidant vitamins from tissue pools and their transfer through the plasma to sites undergoing oxidative stress has been shown to occur during exercise $(3,7)$. These adaptive mechanisms are obviously taking place to combat the increased production of ROS and possible increase of oxidative stress during exercise. The mechanisms behind these adaptive endogenous processes are not well understood. The type of exercise, its intensity and duration are also known to affect antioxidant adaptation (16). Dietary intake of antioxidants is often excluded from consideration in studies observing exercise and oxidative stress, which is a major limitation given the variability of dietary intake between individuals and the significant impact dietary intake has on antioxidant profile (29). Indeed, environmental factors such as exercise and diet can also influence the gene expression of antioxidant enzymes and synthesis of antioxidant molecules $(14,15)$.

The aim of this study was to investigate whether regular exercise training affects oxidative stress or circulating antioxidant defenses during resting conditions in athletes by comparing them to sedentary controls, while also considering dietary intake of antioxidants. Oxidative stress was measured using plasma $\mathrm{F}_{2}$-isoprostane, which has been recognized as the most reliable and sensitive biomarker of lipid peroxidation in body fluids (12). Antioxidant markers measured were plasma total antioxidant capacity; plasma vitamin $\mathrm{E}, \mathrm{C}$, and $\beta$-carotene; plasma urate; erythrocyte SOD and GSHPX.

\section{Methods}

\section{Subjects}

Two groups of subjects were recruited. Exercise-trained athletes were recruited from local track and field athletic clubs who represented the exercise-trained cohort, and age- and sex-matched sedentary controls were recruited from the local university. Volunteers in both groups ranged in age from 18 to 35, were nonsmokers, did not use vitamins, minerals, herbal supplements, or medication (with the exception of contraceptives) and did not report any serious medical conditions. Subjects were included in the athlete cohort if they undertook at least three formal exercise sessions with their athletic club each week. They were a mixture of triathletes, sprinters, and middle distance- and long distance-runners. Control subjects were recruited if they participated in less than $1 \mathrm{~h}$ of planned activity each week. Written informed consent was obtained from all subjects and the study was approved by the Human Research Ethics Committee at the University of Newcastle.

\section{Exercise}

Exercise intensity and duration were quantified (h/wk) using the International Physical Activity Questionnaire (IPAQ)(short, last 7d self-administered format) (6). The IPAQ is comprised of 4 questions that determine the subject's average amount of strenuous and moderate exercise, as well as the amount of walking and sitting they do each day. Exercise had to be at least $10 \mathrm{~min}$ of continuous activity to be recorded. Strenuous exercise is classified as intensities similar to jogging, heavy lifting, digging, aerobics, or fast cycling. Moderate physical activity is classified as 
exercise intensities equivalent to carrying light loads, cycling at a regular pace, or doubles tennis. The walking category included walking at work, at home, to travel from place to place, for recreation, sport, exercise, or leisure. Sitting included sitting at a desk or table, visiting friends, reading, traveling, and watching television.

\section{Energy Requirements}

The Harris-Benedict equation was used to estimate mean daily energy requirements for study subjects (35). It was chosen as it has been shown to be the best energy requirement prediction equation for resting metabolic rate in an athletic population without body composition data (35). The Harris-Benedict equation estimates resting metabolic rate (RMR) which is multiplied by an activity factor to establish mean daily energy requirements. Control's RMR was multiplied by a factor of 1.5 to account for their small level of moderate- and walking-activities and athlete's RMR was multiplied by a factor of 2.1 to represent their additional daily vigorous activities.

\section{Dietary Analysis}

Subject intakes of energy, macronutrient, and known dietary antioxidants, ascorbic acid, and $\beta$-carotene were estimated in this study using 4-d weighed food records, which has been shown to be the most accurate way of assessing dietary intake (4). Subjects were asked to complete the records for 2 working and 2 weekend days. All subjects were given in-depth verbal and written instructions and a practical demonstration of how to weigh and measure food quantities. Subjects were instructed to continue their usual dietary intake during this period. Food records were analyzed using the FoodWorks program (version 2.1, build 146, Xyris Software, Highgate Hill, QLD Australia). Unfortunately, this package does not quantify $\alpha$-tocopherol intake, because of the unavailability of this nutrient on the Australian food composition database. This is a major limitation of the study.

\section{Blood Collection}

All subjects were encouraged to refrain from exercising for at least $24 \mathrm{~h}$ before blood collection. Twenty milliliters of whole blood was collected by venipuncture after a $12 \mathrm{~h}$ overnight fast. Plasma and red blood cell (RBC) pellets were aliquoted into EDTA-coated tubes and stored at $-70^{\circ} \mathrm{C}$. Plasma and RBC pellets were obtained by centrifugation of whole blood at $3000 \times \mathrm{G}$ for $10 \mathrm{~min}$ at $4{ }^{\circ} \mathrm{C}$. Reduced glutathione (1mg/mL blood) (Sigma-Aldrich, St. Louis, MO) was added to the EDTA collection tubes and $200 \mu \mathrm{g}$ butylated hydroxytoluene (BHT) (Sigma-Aldrich) added to tubes used to store plasma for $\mathrm{F}_{2}$-isoprostanes analysis to avoid ex vivo oxidation. All samples underwent only 1 freeze-thaw cycle prior to analysis to avoid ex vivo oxidation or reduction reactions.

\section{Free $F_{2}$-isoprostane (8-iso- $\left.P G F_{2 \alpha}\right)$ Analysis}

To an aliquot of plasma, a known amount of tritiated prostaglandin $\left(\mathrm{PGF}_{2 \alpha}\right)$ (Amersham, Arlington Heights, IL) was added to determine recovery rates. Proteins were precipitated with ethanol, separated by centrifugation at $1500 \times \mathrm{G}$ for $10 \mathrm{~min}$ at 
$4^{\circ} \mathrm{C}$. The supernatant was decanted, ultra pure $\mathrm{H}_{2} \mathrm{O}$ was added to dilute the sample and $\mathrm{pH}$ was lowered via drop-wise addition of hydrochloric acid $(\mathrm{HCl})$ to between 2.5 and 2.75. Samples were first passed through a Sep-Pak C-18 reversed-phase cartridge (Waters Corp., Milford, MA). These were activated with methanol, then $1 \mathrm{mM} \mathrm{HCl}$. The sample was loaded and washed with $1 \mathrm{mM} \mathrm{HCl}$ then heptane and finally eluted with ethyl acetate:heptane (1:1). The samples were further purified using Silica Sep-Pak cartridges (Waters) activated using methanol and ethyl acetate. The sample was loaded and eluted with ethyl acetate:methanol (1:1). The solvents were evaporated under $\mathrm{N}_{2}$ and samples reconstituted in EIA buffer (Cayman Chemical, Ann Arbor, MI). A small amount of sample was then analyzed with an $\mathrm{F}_{2}$-isoprostane enzyme immunoassay (EIA) kit (Cayman). Absorbance values were determined using a plate reader at $405 \mathrm{~nm}$ and the raw data corrected using the recovery rates of tritiated prostaglandin $\left(\mathrm{PGF}_{2 \alpha}\right)$.

The assay was validated by adding known amounts of pure $\mathrm{F}_{2}$-isoprostane standard to aliquots of purified plasma. Analysis with the EIA kit showed a strong correlation $\left(r^{2}=0.99\right)$ with the known amount of pure $\mathrm{F}_{2}$-isoprostane standard (19). The assay has a $100 \%$ cross-sensitivity with $\mathrm{F}_{2}$-isoprostane; $0.2 \%$ each with $\mathrm{PGF}_{2 \alpha}, \mathrm{PGF}_{3 \alpha}, \mathrm{PGE}_{1}$, and $\mathrm{PGE}_{2}$; and $0.1 \%$ with 6-keto- $\mathrm{PGF}_{1 \alpha}$. The detection limit of the assay is $4 \mathrm{pg} / \mathrm{mL}$ (Cayman).

\section{Total Antioxidant Capacity (TAC) Analysis}

The antioxidant capacity of plasma was analyzed using ImAnOx Colorimetric test system for the determination of antioxidative capacity (Immunodiagnostik, Bensheim, Germany) as $\mu \mathrm{mol} / \mathrm{L}$. A defined amount of hydrogen peroxide $\left(\mathrm{H}_{2} \mathrm{O}_{2}\right)$ was added to plasma and the residual amount, after reaction with plasma antioxidants, was determined calorimetrically by an enzymatic reaction. The detection limit of the assay is $130 \mu \mathrm{mol} / \mathrm{L}$. Samples were stored at $-70{ }^{\circ} \mathrm{C}$ for a maximum of $7 \mathrm{~d}$.

\section{Glutathione Peroxidase}

RBC pellets were washed with 10 volumes of cold buffer (50mM TRIS-HCl, $\mathrm{pH}$ 7.5, containing $5 \mathrm{mM}$ EDTA, and $1 \mathrm{mM}$ dithiothreitol), vortexed, centrifuged at $8500 \times \mathrm{G}$ for $10 \mathrm{~min}$ at $4{ }^{\circ} \mathrm{C}$ and the supernatant discarded. Four volumes of cold deionizer $\mathrm{H}_{2} \mathrm{O}$ were added to lyse the resulting $\mathrm{RBC}$ pellet and vortexed. This suspension was then centrifuged at $8500 \times \mathrm{G}$ for $10 \mathrm{~min}$ at $4{ }^{\circ} \mathrm{C}$. The supernatant was collected and stored at $-70^{\circ} \mathrm{C}$. Erythrocyte GSHPx activity $(\mathrm{U} / \mathrm{mL})$ was measured using a GPx-340 colorimetric assay kit (Bioxytech, Oxis International, Inc., Portland, OR). The hemoglobin ( $\mathrm{Hb})$ concentration of the samples was also measured using Sigma-Aldrich kit no. 525 for total hemoglobin, to allow erythrocyte GSHPx activity to be expressed as $\mathrm{U} / \mathrm{mg}$ of $\mathrm{Hb}$.

\section{Superoxide Dismutase}

Four volumes of cold deionized $\mathrm{H}_{2} \mathrm{O}$ were added to the sample, then vortexed. Ice-cold extraction reagent (ethanol/chloroform $62.5 / 37.5[\mathrm{v} / \mathrm{v}]$ ) was added, then vortexed and centrifuged at $3000 \times \mathrm{G}$ for $5 \mathrm{~min}$ at $4{ }^{\circ} \mathrm{C}$. The upper aqueous layer was collected for analysis. Erythrocyte $\mathrm{Zn} / \mathrm{Cu}$ (superoxide dismutase) SOD activity 
(U/mL) was analyzed using a SOD-525 spectrophotometric assay kit (Oxis). The $\mathrm{Hb}$ concentration of the samples was also measured using Sigma-Aldrich kit no. 525 for total hemoglobin, to allow erythrocyte $\mathrm{Zn} / \mathrm{Cu}$ SOD activity to be expressed as $\mathrm{U} / \mathrm{mg}$ of $\mathrm{Hb}$.

\section{Plasma Vitamin ( $E, C$, and $\beta$-carotene) Analysis}

Plasma collected for vitamin $\mathrm{E}, \mathrm{C}$, and $\beta$-carotene analysis was processed and stored at $-70{ }^{\circ} \mathrm{C}$ within $30 \mathrm{~min}$ of blood collection. These samples were stored in the dark at $-70{ }^{\circ} \mathrm{C}$ for a maximum of $5 \mathrm{~d}$ to avoid degradation. Vitamin $\mathrm{A}, \mathrm{E}$, and $\beta$-carotene were separated on a reverse phase high performance liquid chromatography (HPLC) column and measured using a programmable wavelength UV-visible detector (25). Samples were thawed, mixed with ethanol to precipitate proteins, vortexed, and then hexane was added. After vortexing again, samples were centrifuged and the hexane phase removed and injected into an HPLC column (lab-packed Whatman ODS 3 [5 micron] $300 \times 3.5 \mathrm{~mm}$ ID), with a flow rate of 1 $\mathrm{mL} / \mathrm{min}$, run time of $20 \mathrm{~min}$, at ambient temperature. At $0.01 \mathrm{~min}$, vitamin A was measured at $310 \mathrm{~nm}$, at $5.5 \mathrm{~min}$ vitamin E was measured at $280 \mathrm{~nm}$, and at $9.0 \mathrm{~min}$ $\beta$-carotene was measured at $450 \mathrm{~nm}$. Plasma vitamin $C$ was separated on a reverse phase HPLC column and measured using an electrochemical detector (17). Samples were mixed with trichloroacetic acid to precipitate proteins, vortexed, centrifuged, and the supernatant injected into an HPLC column (lab-packed Whatman ODS 3

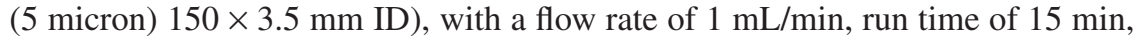
at ambient temperature. Measurements were made with an amperometric electrochemical detector with potential $+0.6 \mathrm{~V}$ versus $\mathrm{Ag} / \mathrm{AgCl}$ reference electrode.

\section{Uric Acid Analysis}

Plasma uric acid (mmol/L) was analyzed using a commercially available assay kit (Roche Diagnostics, Castle Hill, NSW Australia) following standard procedures on a COBAS-BIO centrifugal analyzer (Roche Diagnostics). A reagent containing defined amounts of uricase, N-ethyl-N(2-hydroxy-3-sulfopropyl)-m-toluidine, 4amino-antipyrine, and peroxidase was added to the plasma sample to form a red quinoneimine dye. The color intensity of the quinoneimine formed is directly proportional to the uric acid concentration and is determined by measuring absorbance at $520 \mathrm{~nm}$. Ascorbate oxidase is added to prevent interference by ascorbic acid.

\section{Statistical Analysis}

The statistical evaluation of data collected was done using Minitab version 12 (Minitab, Inc., State College, PA). Comparison of group (control versus athlete) and gender means was analyzed with two-way analysis of variance (ANOVA). One-way analysis of variance was also used to compare differences in groups divided into control, anaerobic- and aerobic athletes. Where significant differences were observed using the one-way ANOVA, post-hoc unpaired $t$-tests were used to evaluate differences within groups. The data are reported as mean \pm standard error of the mean. Pearson's correlation coefficients $(r)$ were used to calculate any linear association between values. Statistical significance was set at $P<0.05$. 


\section{Results}

\section{Subjects}

Table 1 displays the demographic and anthropometric data of the study subjects. Athletes and controls were matched for sex and age. Age matching required the subjects' birth dates to be within $2 \mathrm{y}$ of each other. Two subject pairs fell outside the criteria. The exclusion of these 2 subjects made no difference to the results and they were included in the final data analysis. There were no statistically significant differences in the male or female groups, or when both male and female data were combined, but males were significantly taller and weighed more than female controls and athletes.

\section{Oxidative Stress and Antioxidant Defenses}

No statistically significant differences were observed when groups were separated into controls, aerobic athletes, and anaerobic athletes, except for plasma $\alpha$-tocopherol. Post hoc $t$-tests revealed that significant differences existed separately between controls and the 2 athlete groups, but there was no difference between the athlete groups. Thus, reported data is combined aerobic and anaerobic athletes versus controls. Table 2 presents all oxidative stress and antioxidant defense markers in this study. No statistically significant differences were observed between groups for $\mathrm{F}_{2}$-isoprostane, urate, GSHPx, SOD, or ascorbic acid. Athletes were found to have significantly higher plasma concentrations of $\alpha$-tocopherol and $\beta$-carotene when compared to controls for combined data. Male controls had significantly higher TAC when compared to male athletes, whereas male athletes had higher plasma $\beta$-carotene concentration when compared to male controls. Means of all plasma antioxidants, endogenous enzymes, and TAC were all within normal physiological ranges $(1,21)$.

\section{Energy Requirements and Dietary Intake}

Energy requirements and dietary intakes are displayed in Table 3. Two subjects failed to return their 4-d food records for analysis and a further 5 ( 3 control, 2 athlete) dietary analyses were excluded from statistical analysis because their reported energy intake was less than $1.1 \times \mathrm{BMR}$, which is considered not representative of actual intake (11). Energy intake for athletes was similar to those reported for controls even though estimated energy requirements were significantly higher in athletes. Despite this discrepancy, estimated energy requirements were highly correlated with energy intake $\left(r^{2}=0.541, P<0.001\right)$. Males had significantly higher energy requirements when compared to females. Macronutrient and antioxidant values observed were similar within groups, except for athletes' tendency to have greater carbohydrate intakes and male athletes having a significantly greater intake of ascorbic acid. Macronutrient and antioxidant intakes were all significantly greater in males than in females except for $\beta$-carotene in both controls and athletes and ascorbic acid for controls.

\section{Exercise}

Exercise intensity and duration undertaken by subjects in the week prior to the study is displayed in Table 4 . All athlete groups participated in significantly higher 


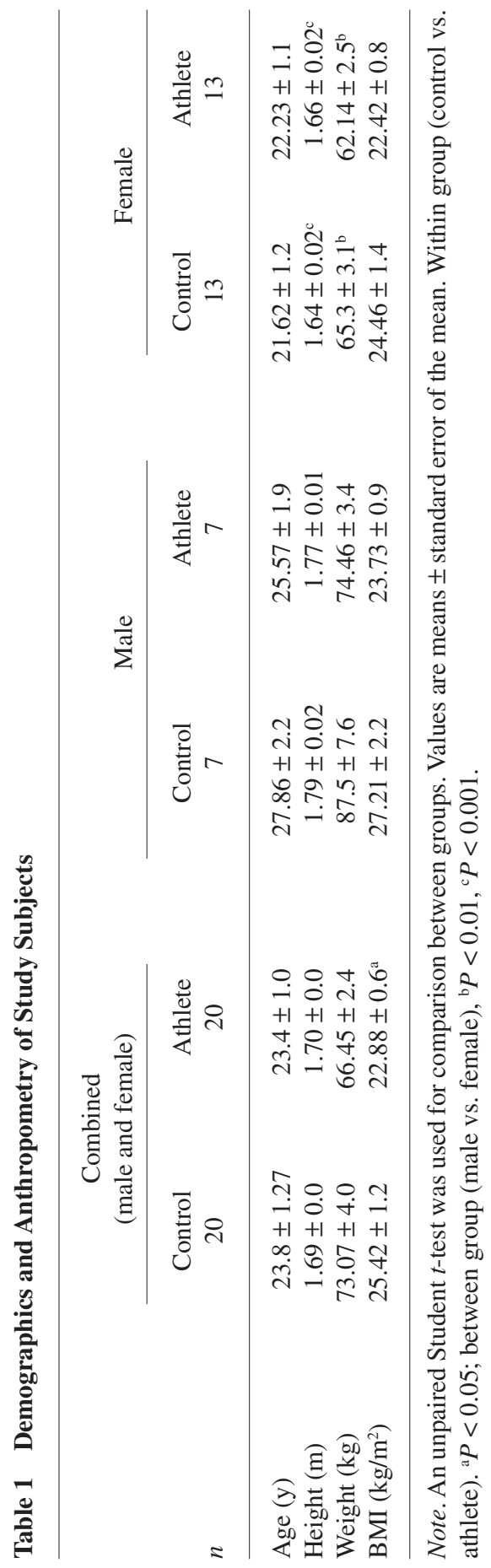




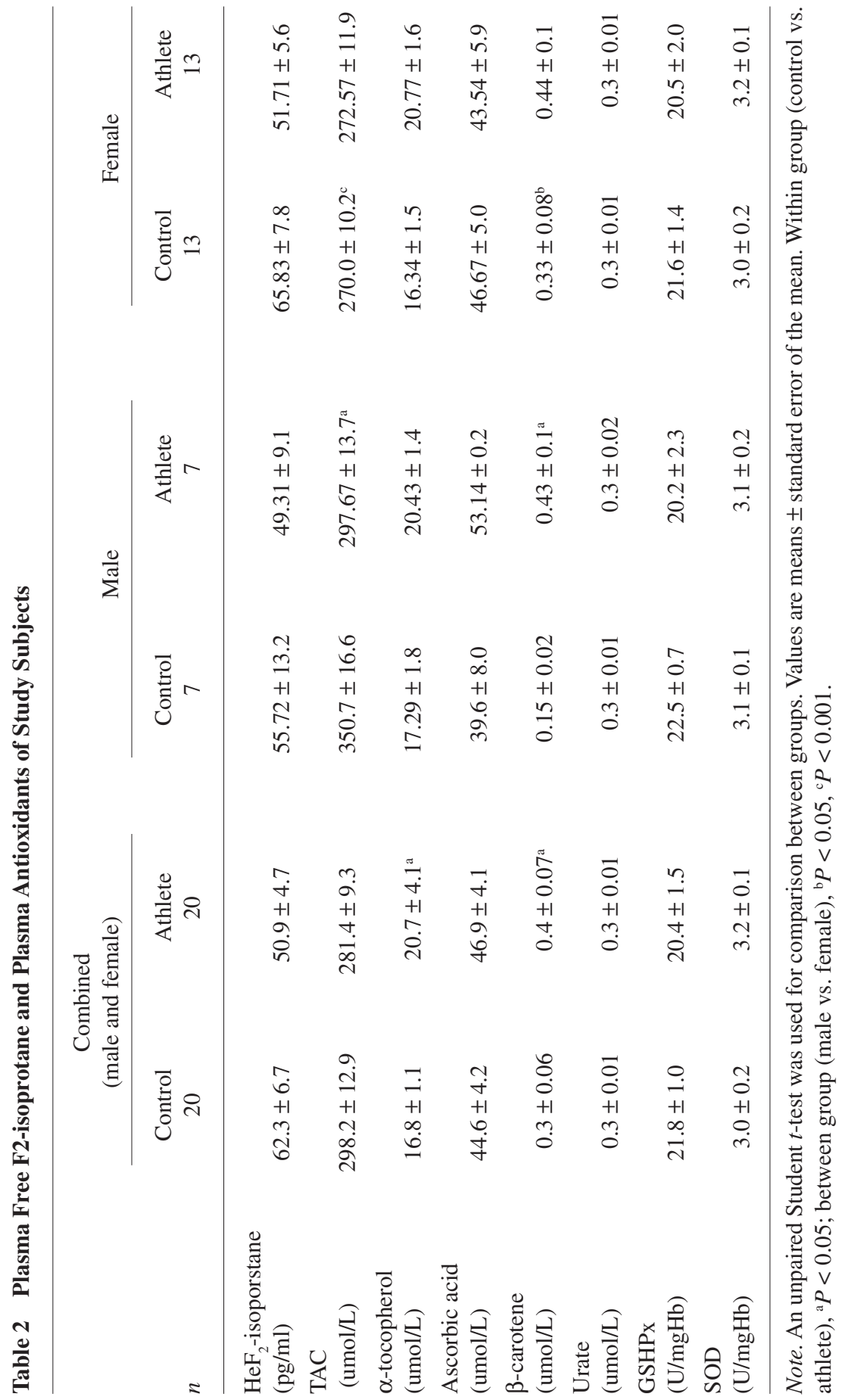




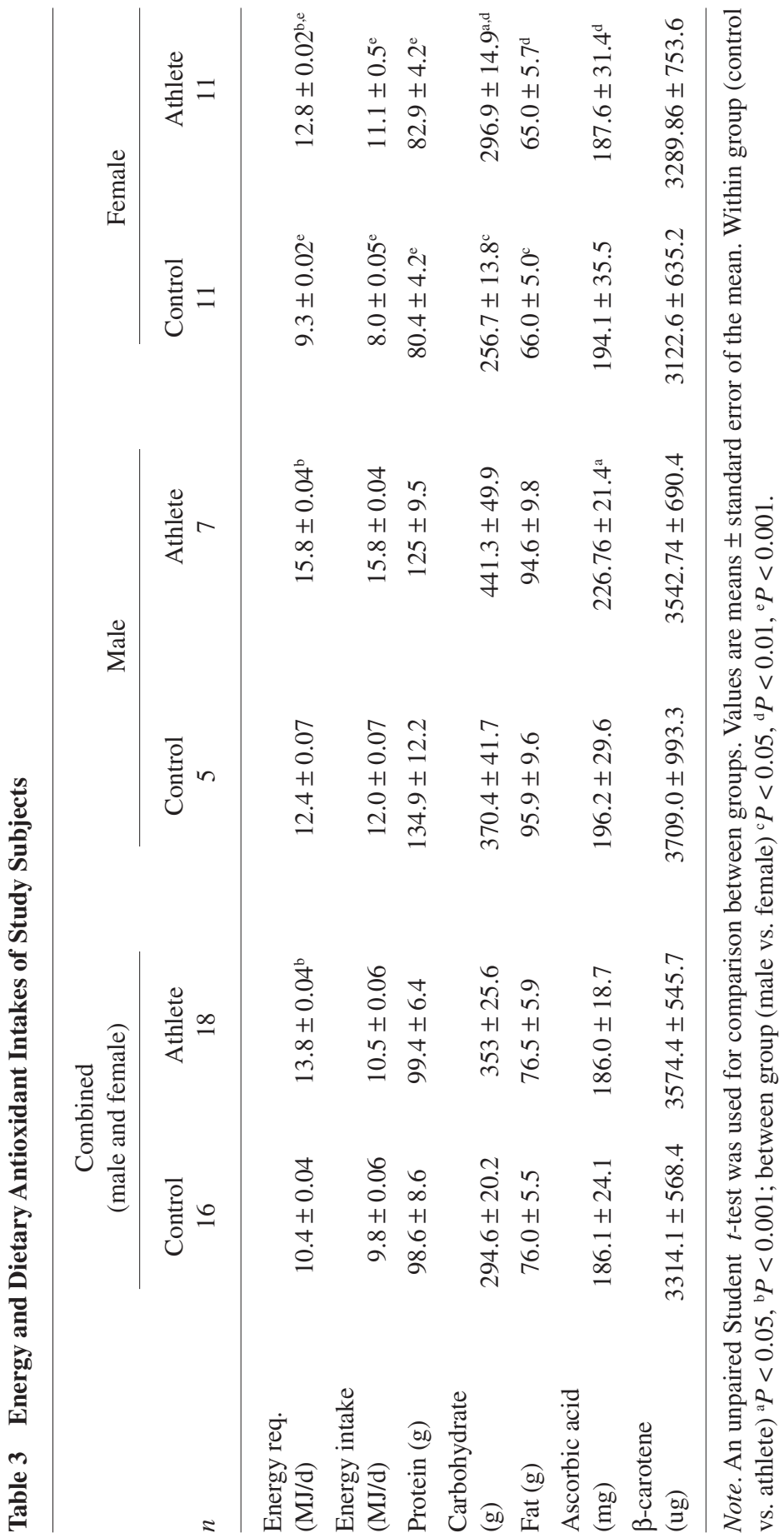




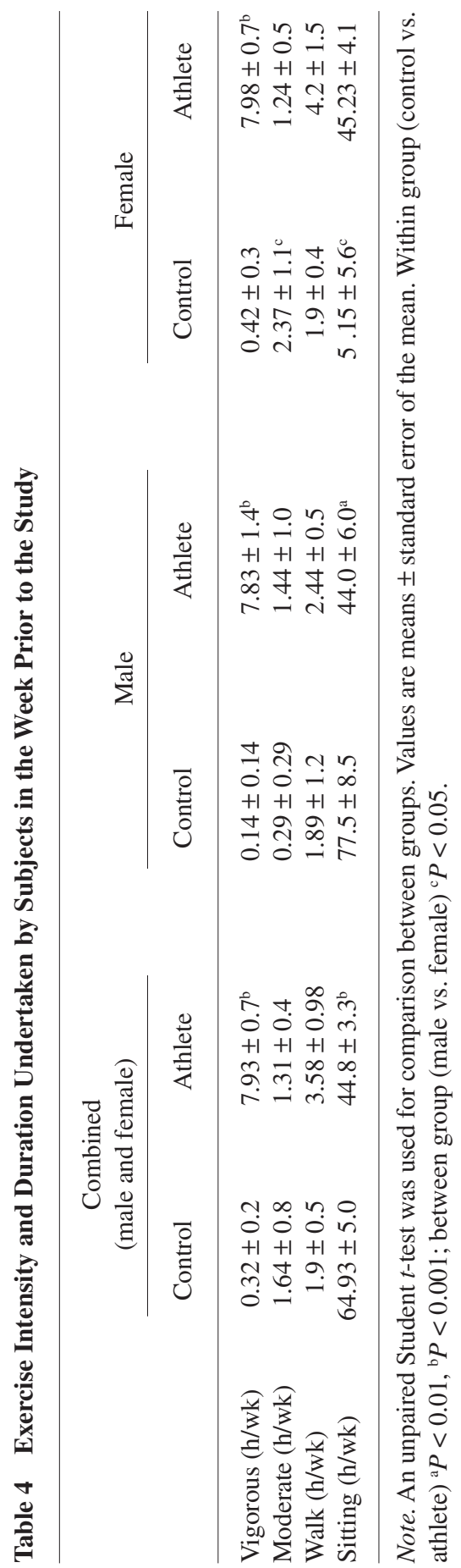


amounts of strenuous activity in the week prior to the study, whereas sedentary controls spent greater time sitting when compared to athletes, which reached significance in the combined and male groups. Female controls did significantly more moderate level exercise and less sitting than the male controls.

\section{Discussion}

The primary aim of this study was to investigate whether regular exercise training affects oxidative stress using $\mathrm{F}_{2}$-isoprostane as a pro-oxidant biomarker or the antioxidant uric acid, glutathione peroxidase (GSHPx), superoxide dismutase (SOD), and plasma total antioxidant capacity (TAC) during resting conditions in athletes and comparing them to sedentary controls. Dietary intake of antioxidants was also considered. The results show that the athlete group, which participated in a significantly greater amount of strenuous exercise on a weekly basis compared to sedentary controls, had similar levels of plasma $\mathrm{F}_{2}$-isoprostane and dietary intakes of antioxidants, but higher circulating concentrations of the antioxidants ascorbic acid and $\beta$-carotene. No up-regulation of endogenous antioxidant enzymes was observed.

Exercise studies $(3,20,30,33)$ that have observed lipid peroxidation biomarkers in athletes under resting conditions and compared them with sedentary controls have used biomarkers (malondialdehyde, thiobarbituric acid reacting substances, conjugated dienes, breath pentane, and ethane) that have been subject to various criticism (8). These studies have returned equivocal results, some showing an increase $(3,20)$ and others a decrease $(30,33)$ in resting lipid peroxidation levels as a result of regular exercise training, which might be attributed to the method of lipid peroxidation analysis (8).

$\mathrm{F}_{2}$-isoprostanes are specific end-products of the nonenzymatic free radical catalyzed oxidation of arachidonic acid (21). It is believed that $\mathrm{F}_{2}$-isoprostanes are formed while still estified to phospholipids in cellular membranes and are released through the action of phospholipases to circulate in free form in body fluid. Thus, any free $\mathrm{F}_{2}$-isoprostanes detected in body fluid arises from cellular membranes and appears a valid marker of lipid peroxidation (9). They are elevated in individuals who engage in activities known to induce oxidative stress such as smoking and acute exercise $(21,24)$; their production is suppressed through antioxidant supplementation (28); they are highly correlated with other lipid peroxidation markers (23) and they do not appear to be confounded by diet (12). Thus, in relative terms, the measurement of $\mathrm{F}_{2}$-isoprostanes is probably the most reliable and sensitive marker of lipid peroxidation in vivo as it has overcome many of the limitations of other lipid peroxidation markers. Not surprisingly, the measurement of $\mathrm{F}_{2}$-isoprostanes in body fluid has been recognized as possibly the most valuable biomarker of lipid peroxidation in a human model (12). Athletes' resting $\mathrm{F}_{2}$-isoprostanes have not previously been compared to sedentary controls. Previous studies, however, have examined $\mathrm{F}_{2}$-isoprostane in athletes during exercise and demonstrated that $\mathrm{F}_{2}$-isoprostane concentration rises transiently with exercise and returns to preexercise levels within 1 to $24 \mathrm{~h}$ recovery $(13,21,34)$. In the present study, athlete $\mathrm{F}_{2}$-isoprostane levels were not found to differ significantly from sedentary controls under resting conditions. There was, however, a tendency for $\mathrm{F}_{2}$-isoprostane to be 
lower in the athlete group (18\%) when compared to sedentary controls and perhaps with a larger sample size statistical significance could have been reached.

The larger than expected inter-subject variation observed in the present study reduced the likelihood of establishing a significant result. Genetic predisposition and the intensity of exercise might be contributory factors for the observed inter-subject variability. Although no significant differences in aerobic $(n=11$; longer duration, moderate intensity) versus anaerobic ( $n=9$; shorter duration, higher intensity) athletes' was observed, there was a tendency for anaerobic athletes to have lower oxidative stress as indicated by reduced $\mathrm{F}_{2}$-isoprostane levels $(P=0.088)$. Several studies using other lipid peroxidation markers have found significantly lower levels in athletes at rest when compared to sedentary controls $(30,33)$. These findings warrant further investigation because lower resting levels of oxidative stress as a result of regular exercise would help explain the paradox of acute exercise increasing oxidative stress but providing protection against chronic disease and enhancing exercise performance. Particular attention needs to be directed to the type, duration, and intensity of exercise influence outcomes.

Elevated antioxidant defenses could explain how lipid peroxidation levels could be lower in athletes. TAC is a marker of the combined capacity of antioxidants in plasma. TAC has previously been shown to be $25 \%$ higher and individual antioxidant markers (ascorbic acid and $\alpha$-tocopherol) have also shown to be higher in trained athletes compared with sedentary controls $(P<0.005)(7)$. In the present study, TAC in athletes was similar to controls except for male athletes who had significantly lower TAC levels which contradicts previous findings. Despite the tendency of TAC to be lower in athletes, plasma $\alpha$-tocopherol and $\beta$-carotene were higher in the athlete groups when compared with controls, which is consistent with previous findings in the literature $(3,7)$. The discrepancy between TAC in the present study with previous findings and the lack of correlation between individual endogenous antioxidants with TAC in this study might be the result of methodological limitations associated with TAC analysis (2). The small contribution of exogenous antioxidants to TAC when compared to other molecules in plasma, such as plasma proteins and urate, could also explain the lack of correlation between endogenous antioxidants and TAC (37). Even with the discrepancy between TAC and individual plasma antioxidant vitamins, higher plasma antioxidant vitamins should provide protection against oxidative stress in the athletes undertaking regular exercise in the present study.

There is growing evidence of an association between obesity and increased oxidative stress and decreased antioxidant defenses (36). In the present study, greater body weight or adiposity, represented by BMI, could possibly provide one plausible reason for the athletes having significantly higher plasma $\alpha$-tocopherol and $\beta$-carotene and a tendency of lower $\mathrm{F}_{2}$-isoprostanes. It is likely that the athlete group maintain a lower BMI because the majority of them were endurance athletes who tend to be lean as result of their significantly greater participation in endurance exercise.

Mobilization of antioxidant vitamins from tissue pools and their transfer through the plasma to sites undergoing oxidative stress is a well-established phenomenon and might serve as a mechanism to explain the increases in athletes' plasma antioxidant vitamins during rest (3). It has been proposed that mobilization of antioxidants is a transient event that occurs during acute exercise and returns 
to preexercise levels with rest (26). Findings from previous studies (7) and those from the present study, however, suggest that mobilization could also account for a more chronic increase in the baseline circulation of plasma antioxidants in those exercising regularly. The antioxidants mobilized during intense exercise might spillover during recovery into rest periods and account for the findings in the present study.

It is well recognized that increased intakes of dietary vitamins are generally reflected through higher concentrations in plasma, which could also explain the tendency for athletes to have higher resting plasma levels of antioxidant vitamins (12). Dietary analysis in the present study indicates that antioxidant intakes for both groups were similarly high. Both groups mean daily intake of ascorbic acid was more than 4 times the Recommended Dietary Intake (RDI) for Australians $(40 \mathrm{mg} / \mathrm{d})$ and intake of $\beta$-carotene was higher than that reported in the Australian National Nutrition Survey (NNS) for males and females between 19 and 24 y (22). The NNS survey was used as a means of comparison as $\beta$-carotene does not have an RDI because it is not considered an essential nutrient. Unfortunately, $\alpha$-tocopherol intake could not be quantified in the present study as it is not included in Australian food databases. The absorption and utilization of $\alpha$-tocopherol depends substantially on the presence of dietary lipids, particularly polyunsaturated fatty acids (PUFAs). Because there was no significant difference between groups for dietary fat intake it is unlikely that dietary $\alpha$-tocopherol would be different between the 2 groups (31).The similarity in antioxidant intake between groups excludes dietary intake from being a direct mechanism explaining increased plasma antioxidant vitamins; however, it could contribute indirectly through mobilization. The body's ability to mobilize antioxidants from tissue stores and transport them through plasma would also suggest that plasma levels of antioxidants are not reflective of tissue levels or overall antioxidant status in athletes, unless that athlete is deficient in an antioxidant. It is also noteworthy that to mobilize $\alpha$-tocopherol and $\beta$-carotene from tissue stores they must be initially present. $\alpha$-tocopherol and $\beta$-carotene are nutrients that can only originate from the diet, highlighting the importance of adequate dietary intake of antioxidant nutrients. Alternatively, $\alpha$-tocopherol and $\beta$-carotene might be spared based on other antioxidant availability.

Up-regulation of erythrocyte antioxidant enzymes as a result of exercise training could account for lower resting oxidative stress levels observed in the present study. The literature reports equivocal results regarding the response of erythrocyte enzymes to regular exercise training. SOD activity has been found to remain unaltered $(30,33)$ or increased $(3,7,20)$. GSHPx activity has been found to remain unaltered (33), increase $(20,30,33)$, and decrease (3). In the present study, no up-regulation of SOD or GSHPx was evident at rest in athletes when compared with controls, which excludes it from influencing $\mathrm{F}_{2}$-isoprostane concentrations in the athlete groups. Differences in athlete antioxidant intakes, the type of exercise, its intensity, and duration at training are all factors that have been shown to influence endogenous antioxidant enzymes and might explain the discrepancies between studies $(16,36)$.

In this study, resting concentrations of the plasma antioxidants $\alpha$-tocopherol and $\beta$-carotene were elevated in those participating in regular strenuous exercise training when compared to sedentary controls. No difference in the dietary intake of antioxidants was observed. Thus, greater body weight or adiposity of controls 
maintained via less physical activity is one possible explanation for the elevation in plasma $\alpha$-tocopherol and $\beta$-carotene. Antioxidants mobilized during intense exercise and their spillover during recovery into rest periods is another plausible mechanism that could account for the findings in the present study. These 2 mechanisms could explain why increased antioxidant defenses and improved protection against oxidative stress was observed in athletes at rest and might explain part of the health benefit of regular exercise and is an area worthy of further investigation. Future research should consider inter-subject genetic variation, as well as the influence of the type of exercise, its intensity and duration.

\section{Acknowledgments}

Technical assistance received from Robert Blake is gratefully acknowledged. We are also grateful to Hans Tiller for his help recruiting the training volunteers.

\section{References}

1. Abuja, P.M., and R. Albertini. Methods for monitoring oxidative stress, lipid peroxidation and oxidation resistance of lipoproteins. Clin. Chim. Acta. 306:1-17, 2001.

2. Alessio, H. M., A.H. Goldfarb, and G. Cao. Exercise-induced oxidative stress before and after vitamin C supplementation. Int. J. Sport Nutr. 7:1-9, 1997.

3. Balakrishnan, S.D., and C.V. Anuradha. Exercise, depletion of antioxidants and antioxidant manipulation. Cell Biochem. Funct. 16:269-275, 1998.

4. Bingham, S.A., A. Cassidy, T.J. Cole, A. Welch, S.A. Runswick, A.E. Black, D. Thurnham, C. Bates, K.T. Khaw, T.J. Key, et al. Validation of weighed records and other methods of dietary assessment using the $24 \mathrm{~h}$ urine nitrogen technique and other biological markers. Br. J. Nutr. 73:531-550, 1995.

5. Bishop, N.C., A.K. Blannin, N.P. Walsh, P.J. Robson, and M. Gleeson. Nutritional aspects of immunosuppression in athletes. Sports Med. 28:151-176, 1999.

6. Booth, M. Assessment of physical activity: an international perspective. Res. Q. Exerc. Sport. 71:S114-S120, 2000.

7. Brites, F.D., P.A. Evelson, M.G. Christiansen, M.F. Nicol, M.J. Basilico, R.W. Wikinski, and S.F. Llesuy. Soccer players under regular training show oxidative stress but an improved plasma antioxidant status. Clin. Sci. (Lond.). 96:381-385, 1999.

8. Clarkson, P.M., and H.S. Thompson. Antioxidants: what role do they play in physical activity and health? Am. J. Clin. Nutr. 72:637S-646S, 2000.

9. Cracowski, J.L., T. Durand, and G. Bessard. Isoprostanes as a biomarker of lipid peroxidation in humans: physiology, pharmacology and clinical implications. Trends Pharmacol. Sci. 23:360-366, 2002.

10. Davies, K.J., A.T. Quintanilha, G.A. Brooks, and L. Packer. Free radicals and tissue damage produced by exercise. Biochem. Biophys. Res. Commun. 107:1198-1205, 1982.

11. Goldberg, G.R., A.E. Black, S.A. Jebb, T.J. Cole, P.R. Murgatroyd, W.A. Coward, and A.M. Prentice. Critical evaluation of energy intake data using fundamental principles of energy physiology: 1. Derivation of cut-off limits to identify under-recording. Eur. J. Clin. Nutr. 45:569-581, 1991.

12. Halliwell, B. Establishing the significance and optimal intake of dietary antioxidants: the biomarker concept. Nutr. Rev. 57:104-113, 1999. 
13. Hinchcliff, K.W., G.A. Reinhart, R. DiSilvestro, A. Reynolds, A. Blostein-Fujii, and R.A. Swenson. Oxidant stress in sled dogs subjected to repetitive endurance exercise. Am. J. Vet. Res. 61:512-517, 2000.

14. Jackson, M. J. Free radicals in skin and muscle: damaging agents or signals for adaptation? Proc. Nutr. Soc. 58:673-676, 1999.

15. Jackson, M.J., A. McArdle, and F. McArdle. Antioxidant micronutrients and gene expression. Proc. Nutr. Soc. 57:301-305, 1998.

16. Ji, L.L. Antioxidants and oxidative stress in exercise. Proc. Soc. Exp. Biol. Med. 222: 283-292, 1999.

17. Lee, W., P. Hamernyik, M. Hutchinson, V.A. Raisys, and R.F. Labbe. Ascorbic acid in lymphocytes: cell preparation and liquid-chromatographic assay. Clin. Chem. 28: 2165-2169, 1982.

18. Leeuwenburgh, C., and J.W. Heinecke. Oxidative stress and antioxidants in exercise. Curr. Med. Chem. 8:829-838, 2001.

19. MacDonald-Wicks, L.K., and M. L. Garg. Modulation of carbon tetrachloride-induced oxidative stress by dietary fat in rats. J. Nutr. Biochem. 13:87-95, 2002.

20. Marzatico, F., O. Pansarasa, L. Bertorelli, L. Somenzini, and G. Della Valle. Blood free radical antioxidant enzymes and lipid peroxides following long-distance and lactacidemic performances in highly trained aerobic and sprint athletes. J. Sports Med. Phys. Fitness. 37:235-239, 1997.

21. Mastaloudis, A., S.W. Leonard, and M.G. Traber. Oxidative stress in athletes during extreme endurance exercise. Free Radic. Biol. Med. 31:911-922, 2001.

22. McLennan, W., and A. Prodger. National Nutrition Survey: Nutrient Intakes and Physical Measurements. Australia. 1995. Canberra: Australian Bureau of Statistics, 1998, 24-25.

23. Meydani, M. Isoprostanes as oxidant stress markers in coronary reperfusion. Nutr. Rev. 55:404-407, 1997.

24. Morrow, J.D., B. Frei, A.W. Longmire, J.M. Gaziano, S.M. Lynch, Y. Shyr, W.E. Strauss, J.A. Oates, and L.J. Roberts, 2d. Increase in circulating products of lipid peroxidation (F2-isoprostanes) in smokers. Smoking as a cause of oxidative damage. N. Engl. J. Med. 332:1198-1203, 1995.

25. Nilsson, B., B. Johansson, L. Jansson, and L. Holmberg. Determination of plasma alpha-tocopherol by high-performance liquid chromatography. J. Chromatogr. 145: 169-172, 1978.

26. Pincemail, J., C. Deby, G. Camus, F. Pirnay, R. Bouchez, L. Massaux, and R. Goutier. Tocopherol mobilization during intensive exercise. Eur. J. Appl. Physiol. Occup. Physiol. 57:189-191, 1988.

27. Powers, S.K. and K. Hamilton. Antioxidants and exercise. Clin. Sports Med. 18:525536, 1999.

28. Pratico, D., R.K. Tangirala, D.J. Rader, J. Rokach, and G.A. FitzGerald. Vitamin E suppresses isoprostane generation in vivo and reduces atherosclerosis in ApoE-deficient mice. Nat. Med. 4:1189-1192, 1998.

29. Record, I.R., I.E. Dreosti, and J.K. McInerney. Changes in plasma antioxidant status following consumption of diets high or low in fruit and vegetables or following dietary supplementation with an antioxidant mixture. Br. J. Nutr. 85:459-464, 2001.

30. Robertson, J.D., R.J. Maughan, G.G. Duthie, and P.C. Morrice. Increased blood antioxidant systems of runners in response to training load. Clin. Sci. (Lond.). 80:611-618, 1991. 
31. Sacheck, J.M., E.A. Decker, and P.M. Clarkson. The effect of diet on vitamin E intake and oxidative stress in response to acute exercise in female athletes. Eur. J. Appl. Physiol. 83:40-46, 2000.

32. Schwemmer, M., B. Fink, R. Kockerbauer, and E. Bassenge. How urine analysis reflects oxidative stress—nitrotyrosine as a potential marker. Clin. Chim. Acta. 297:207-216, 2000.

33. Selamoglu, S., F. Turgay, B.M. Kayatekin, S. Gonenc, and C. Yslegen. Aerobic and anaerobic training effects on the antioxidant enzymes of the blood. Acta Physio.l Hung. 87:267-273, 2000.

34. Steensberg, A., J. Morrow, A.D. Toft, H. Bruunsgaard, and B.K. Pedersen. Prolonged exercise, lymphocyte apoptosis and F2-isoprostanes. Eur. J. Appl. Physiol. 87:38-42, 2002.

35. Thompson, J., and M.M. Manore. Predicted and measured resting metabolic rate of male and female endurance athletes. J. Am. Diet. Assoc. 96:30-34, 1996.

36. Vincent, H.K., J.W. Morgan, and K.R. Vincent. Obesity exacerbates oxidative stress levels after acute exercise. Med. Sci. Sports Exerc. 36:772-779, 2004.

37. Wayner, D.D., G.W. Burton, K.U. Ingold, L.R. Barclay, and S.J. Locke. The relative contributions of vitamin E, urate, ascorbate and proteins to the total peroxyl radicaltrapping antioxidant activity of human blood plasma. Biochim. Biophys. Acta. 924 : 408-419, 1987. 